EDITED FOR THE ASSOCIATION BY N. S. DAVIS.

PUBLISHED WEEKLY.

Vol. V.

Chicago, August 29, 1885.

No. 9 .

ORIGINAL ARTICLES.

\section{THE SIMILARITY OF ELECTRIC AND NERVE FORCES. ${ }^{1}$}

BY JOHN J. CALDWELI, M.D., OF BALTIMORE, MD.

In the torpedo the body is somewhat oval and rounded. Our native species, found mostly in winter, especially on the low, sandy shores of Cape Cod, is Torpedo occidentalis (Storer). Its batteries and nerves are substantially as in the European species. The electrical organs are constructed on the principle of the Voltaic pile, consisting of two series or layers of hexagonal cells, the space between the numerous fine transverse plates in the cells filled with a jelly-like, trembling mass, each cell representing, so to speak, a Leyden jar. There are about 470 cells in each battery, each provided with nerves sent off from the fifth and eighth pairs of nerves. The dorsal side of the apparatus is positively electrical, the ventral side negatively so. The electrical current passes from the dorsal to the ventral side. When the electrical ray is disturbed by the touch of any object, the impression is conveyed by the sensory nerves to the brain, exciting there an act of will which is conveyed along the electric nerves to the batteries, producing a shock. The benumbing force is lost by frequent exercise, being regained by rest; it is also increased by energetic circulation and respiration. As in muscular exertion, the electrical power is increased by the action of strychnine. (Owen.)

Marey has quite recently made interesting experiments on the torpedo, examining the discharge of the fish with the telephone. Slight excitations provoked a short, croaking sound. Each of the small discharges was composed of a dozen plexus and pulsations, and lasting about one-fifteenth of a second. The sound from a prolonged discharge, however, continued three or four seconds, and consisted of a sort of groan, with a tonality of about $m i$ ( 65 vibrations), agreeing pretty closely with the result of "graphic experiments." Marey has also studied the resemblance of the electrical apparatus of the electrical ray or torpedo and a muscle. Both are subject to will, provided with nerves of centrifu-

1Read in the Section of Practical Medicine, Materia Medica and Physiology at the 'Ihirty-Sixth Annual Meeting of the American Medical Association. gal action, have a very similar chemical composition, and resemble each other in some points of structure. A muscle in contraction and in tetanus executes a number of successive small movements or shocks, and a like complexity has been proved by M. Marey in the discharge of the torpedo.-(Packard, Zoölogy.)

Malapterurus electricus (Lacepede), of the Nile, is electrical, the electric cells forming a layer directly beneath the skin, and enveloping the whole body except the head and fins. The cells are minute, lozenge-shaped, about one and a half millimetres in diameter. They are supplied by a nerve from the spinal cord. The shock is comparatively feeble, but suffices for defence, the fish being protected by its electrifying coat as the hedge-hog by its spines. (Owen.) One of the lowest teleosts, the electrical eel (gymnotus electricus), of South America, is two metres in length, and is characterised by its greatlydeveloped electrical batteries. These are four in number, situated two on each side of the body, and together form nearly the whole lower half of the trunk. 'The plates of the cells are vertical instead of horizontal as in the torpedo, while the entire batteries, or cells, are horizontal instead of vertical as in the electrical ray. The nerves sent to the batteries of the eel are supplied by the ventral branches of about two hundred pairs of spinal nerves.

In the torpedo there are about 470 cells in each battery. This is one of the lowest orders of vertebrates, and therefore the battery construction is simple, the discharges resembling more those from a charged Leyden jar. The machine remains at rest to be re-charged before another manifestation of power. As we ascend, the organism becomes more complex, and in the higher fishes and reptilia and lower mammals the electric apparatus resembles more dynamic electricity, whilst in chimpanzee and gorilla we have Faradic electricity; and, lastly, in man the phenomena require a combination of the different manifestations of this force. Hence we have discharge as from a Leyden jar when we knock a man heels over head; whilst in reasoning we employ, or bring into relation, or co-ordinate all of the different arrangements, resulting in magneticostatico-dynamic force-ratiocination. (S.)

As I have contended for years past, it is apparent, from the close analogy of electricity to nerve force, that this agent embraces a wide range of morbid conditions. Through the nervous cords which act as conductors, every part of the animal economy can be reached. In this way sccretion and elimination 
of morbid products may be promoted, and the organ or apparatus restored to healthy action. 'The three great forces of nature are heat, light, and electricity. These are the forces under whose influence vegetation is produced. Heat and moisture cause the germ of life in seed to awaken; the materials stored up for the use of the embryo undergo change, both chemical and mechanical, so as to be fit for appropriation. The architecture of the plant begins. A spire shoots up from the bud, under the influence of the sun's light; the food afforded by the atmosphere is appropriated. Forces are active at the root; forces are active in the blade. 'There is no doubt, in my opinion, that electricity is one of the active forces which contribute to plant growth. We have all the conditions of its generation-heat, moisture, unequal heating of different mineral substances, causing thermo-electricity. We also have chemical action. It is impossible to draw a line of demarcation between vegetable and animal structure, and I doubt not that heat, light and electricity are the physical forces under which animal structure is built up. The light of the sun is as necessary to the health and vigor of the animal as of the vegetable kingdom. Indeed, as already stated, the two shade into each other so as to render it impossible to say where the regetable ceases and the animal begins. Electricity, being one of the forces which contribute to the animal organism, is necessary to functional health.

EIECTRICAL FORCE IN MAN AND NATURE.

All phenomena of the organic or inorganic world are. now being interpreted in the terms of matter, motion and force. The pilot idea of the doctrine of evolution to-day is that everything proceeds from the simple to the complex, and that back of all the manifestations of matter and of mind there lies "unity of force." It is this acting, living, persistent principle which constantly sits at the loom of time, and arranges the position and the texture of the threads that make up the web of things. This ancient weaver called "Force" has received many different names. When it works among molecules we call it cohesive attraction. When it works among the ultimate atoms, building them up into the delicate and beautiful crystals we call it the crystallizing forces. When engaged in uniting kindred atoms we call it chemical force, or chemical affinity. Our meaning, however, is sufficiently indicated without further extension of the list of forces.

Nature delights in prodigality of results, but she is parsimonious in the number of her working powers. Given a little matter and motion and she will construct a world. The lines of force radiate in all directions, always acting, always persistent. Difference of result is but the product of the same force differently conditioned. A tree and a mammal betray the same parent force. In the ultimate analysis we reach simplicity of cause and "unity of force."

Perhaps one of the most impressive instances of the play of force is to be seen in the phenomena of light. Light is but a result of the rapid oscillations of the ether waves. Hundreds of millions of these waves strike the retina before we are conscious of the sensation of light. How this sensation is translated into consciousness is a mode of force of which we know nothing. One fact, however, has been determined, viz. : that color is a result of motion. Less rapid oscillations are required to produce in us the sensation of the red in the spectrum than o the violet. Electric light, which is the most intense of all light, is but a product of exalted motion. A certain degree of motion produces sound; this motion increased produces heat; a further increase produces all the colors of the spectrum in their regular order. Still exalting this motion, we obtain electric light.

The theory of wave motion in the ether had its origin in the wave motion of water and atmosphere. The ear sustains the same relation to sound that the eye does to light. There is a close analogy between the harmony of sound and the harmony of color. The rhythm of sound has its unvarying conditions in reference to the human ear. Atmospheric vibrations, when they exceed a certain number per second, lose their individuality, and become merged into a hum of indistinct sound. The auditory nerve is finely adapted to take in the whole range of the gamut. Sounds, higher or lower, in either direction, cease to be distinct.

It remains to say a word in reference to the more special manifestations of force. The human system supplies the most appropriate example. From the persistence of force we can but conclude that between the mind and body there is the closest relation, sensation, thought, and our most involved sentiments imply the destruction of waste of the gray tissue of the brain molecules. This waste is only another name for one mode of the "play of force." In a word, man, as he is, is but a product of force, acting, in the first instance, on a germ hardly microscopic, and mind, with all its wonderful and complicated powers, is evolved from the simplest beginnings. A Raphael or a Newton are evolved, in common with the lowest orders of animal life, from the product of the same force, controlled and governed by the same law, which had its fitting conditions and relations. Want of harmony in these, as we before remarked, implies want of harmony in result. A healthy mind, healthy morals and a healthy religion are the resultants of the unimpeded action of force.

\section{THE ADVANCE OF MEDICAL SCIENCE.}

A knowledge of the brain and of the network of the nervous system is the essential to every educated physician, who would minister to a body, or to a mind diseased. The ability to maintain a proper medium, through the exaltation or depression of nerve power is requisite to him who would understand correctly the nervous system. Her path to universal empire, will continue to be, as it is now, dotted all along with rejected theories. She is now making rapid strides, and has indeed become an ocean of truth. Let us keep abreast, at least, o that portion of its mighty tide which is now carrying the science of medicine beyond its ancient land- 
marks, enriching it with new truths. Just how elec- mo-electricity. We also have chemical action. It is tricity acts as a therapeutic agent, no experimenter impossible to draw a line of demarcation between has satisfactorily explained, though learned minds vegetable and animal structure, and doubt not that have elaborated ingenious theories, simple and com- heat, light and electricity are the physical forces unplex; still we will maintain our text: "The unity of der which animal structure is built up. 'The light of Force." We will again note that electricity is but the sun is as necessary to the vigor and health of the another name for the most exalted motion known. animal, as of the vegetable kingdom. Indeed, as alWe will then be able, in some degree, to comprehend ready stated, the two shade into each other so as to its action upon our bodies, and its true place in med- render it impossible to say where the vegetable icine, and how it acts through the brain, and its infi- ceases, and the animal begins. Electricity being nite ramifications. Nerve velocity is 60 yards per second; sound velocity is $33^{2}$ yards per second; cannon ball velocity is $55^{\circ}$ yards per second; light velocity is $360,000,000$ yards per second; electrical velocity is $450,000,000$ yards per second.

Hence it can be readily understood, how electricity, direct action, may accelerate our natural forces; how its reverse action may depress them, how its chemical action may dissolve or electrolize morbid growth; how even may dilate the vascular system, or accelerate the vaso-motor; and how, through the sympathetic system exalt the trophic powers, or produce inhibitory action.

The attention of electricians was concentrated upon Electro-Physics until the perfection of the mechanical contrivance by Morse. In due time it was known to the medical profession that electricity was a powerful therapeutic agent, and its applications in the treatment of diseases wrested from the hands of quacks and charlatans. Electro-Therapy is now an important specialty in medicine. The close analogy of electric to nerve force at once caused attention to be directed to its employment in the treatment of nervous diseases; but experiment has shown that it is applicable to the treatment, the relief and cure of other pathological conditions. Thus it may be used as a tonic in cases of nervous debility, in cases of paralysis so as to take the place of nerve force which presides over functions of nutrition. The nutrition of the paralysed limb is thus sustained, and likewise the revival of nervous energy promoted. It is apparent from the close analogy of electricity to nerve force, that this agent embraces a wide range of therapeutic conditions. Through the nervous cords which act as conductors, every part of the animal organism can be reached. In this way secretion and elimination of morbid products may be promoted, and the organ or apparatus restored to heaithy action.

The three great forces of nature are heat, light and electricity. These are the forces under whose influence vegetation is produced. Heat and moisture cause the germ of life in seed to waken, the materials stored up for the use of the embryo undergo changes both chemical and mechanical, so as to be fit for appropriation. The architecture of the plant begins. A spire shoots up from the bud; under the influence of the sun's light, the food afforded by the atmosphere is appropriated. Forces are active at the root, and forces are active in the blade. There is no doubt that electricity is one of the active forces which tl contributes to plant growth. We have all the conditions of its generation, heat, moisture, unequal heating of different mineral substances, causing therone of the forces which contributes to the animal organism, is necessary to functional health.

The various conditions under which electricity is manifested enable us to apply it to a variety of morbid conditions. Its wonderful decomposing power (electrolysis) has been utilized by the physician. The intense heat incident to its manifestations by certain combinations, has enabled the surgeon to substitute in many cases the cautery for the knife, indeed to apply it to the section of morbid growths not remedial by the knife.

It may be stated in order that progress shall be had in any branch of science, appropriate apparatus is necessary. Scientific speculation is a great lever, but it must be controlled by experiment; speculation must be tested by experiment, for the human mind is too apt to be seduced by the pleasure of speculative philosophy, and too prone to abandlon the paths of observation and sound induction. From the want of apparatus and the ignorance of its different modes of manifestation, electricity as a therapeutic agent was seized upon by the charlatan and neglected by intelligent practitioners of medicine. By a curious coincidence the discoveries of electric science and improved apparatus was accompanied by great discoveries in regard to the functions of the nervous system. Whilst Oersted, Ampère, Faraday and others were making rapid strides in electric science, Sir Charles Bell, Magendie, Marshall Hall, Claude Bernard, Brown-Séquard and others were unravelling the intricacies of the nervous system. In no era of the world has there ever been such activity in scientific investigations, and the applications of science to the material comfort and welfare of mankind. At last the votaries of medicine contribute their quota, and many of the most eminent of the profession are devoting their talents and energies to the therapeutical effects of electric force in the treatment of diseases.

Electricity in suspended rital functions.-We have been induced to try the effects of electricity in cases of suspended vital function resulting from narcosis, and cases of apparent death from drowning, from experiments upon living rabbits, made by Sir Wilson Phillips, of England, from which it appears that electric force was used to substitute nerve power. The pneumogastric nerve of the animal was severed immediately after it had eaten some parsley. After the death of the animal, which had great difficulty of breathing, and perished apparently from suffocation, the stomach was opened and the parsley found undigested. A similar experiment of section of pneumogastric nerve was made, but a current of galvanism passed along the nerve, and continued for 26 hours. 
No difficulty of breathing occurred as long as the current was kept up. The animal was killed, the stomach examined, the parsley was'completely digested. It thus appeared that galvanic energy is capable of supplying nervous influence in that the pro cess of digestion may be carried on. Similar experiments were made upon dogs with like results.

Influence of Ancesthesia on Vaso-motor Forces of the Circulation.-The following experiments by Drs. Bowdich and Minot illustrate the influence of anrsthetics on vaso-motor centres.

Anæsthetics, in producing insensibility, have accomplished such results by antagonizing the effects of irritation of sensitive nerves. One of the most constant physiological results of irritation of a sensitive nerve is a rise of arterial tension, due to a reflex stimulation through the vaso-motor centres of the muscular walls of the smaller arteries, especially those of the intestines. It is ascertained that in the majority of cases, the rise of blood tension consequent upon the irritation of the saphenous nerves, is less marked when the animal is under the influence of ether, than when the anæsthetic is not used. The first object was to determine the effects of anxesthesia on the reflex rise of the blood tension. This was accomplished in the following manner: An animal being placed on the operating table in the supine position, a solution of curari was injected into the jugalar vein, when paralysis ensued. When the respiratory movement ceased, the trachea was connected by means of a glass canula inserted into it with the apparatus for artificial respiration, which was so adjusted as to imitate as closely as possible the normal respiratory rhythm. A canula was then placed in the carotid artery and connected with a mercury manometer, carrying a pen by means of which the blood tension was recorded on a long strip of paper, which was kept in uniform motion by clock-work. The saphenous nerve was then placed upon electrodes. The irritation of the nerve was produced by closing the currents, by means of a key provided with a pen, thus recording the blood tension, which could be seen at a glance. After the anæsthetic had been administered the nerve was again irritated. Then the blood tension was notably decreased, and so continued to be as long as these experiments were tried.

But far more constant and obvious were the results obtained from chloroform. Here the irritation of the saphenous nerve caused a less marked rise in the blood tension, and sometimes there was no rise whatever. These facts present to my mind the clearest evidence in favor of the electrical remedy in cases of deep chloroform toxæmia, and the propriety of having accessibility to a Faradic instrument, complete and ready for immediate use in chloroform administrations.

E. Du Bois-Reymond, "first succeeded in demonstrating the presence of specific muscle and nerve currents by deflection of the magnetic needle." $\mathrm{He}$ likewise ascertained that, "if the muscle or nerve be excited by electric currents, or by mechanical or chemical irritants, so that the first is physiologically active, and the latter caused to contract, and then placed at two symmetrical points in connection with the galvano-multiplier, a less deflection of the needle is produced than when the nerve or muscle is in a quiescent state. This is called the negative variation of the current." The conclusion arrived at by Du Bois-Reymond was, that nerve and muscle contain innumerable positive and negative electric molecules which move with great regularity throughout the tissue. Perchance the power of electricity in cases of suspended animation is to restore the suspended electrical forces of the body to a normal condition, and thus reanimate failing vitality.

The effect of chloroform, the resuscitation of the still-born, and of the tranced, and of the inert uterus in post partum hæmorrhage, may be remedied in much the same way; that is to say, by appealing to the vaso-motor centres through the pneumogastric axis.

No nervous centre can act of its own motion or originate action. It must be excited to action. We might as well speak of sight without the action of the waves of the luminous ether, or sound without the vibration of sonorous substances. In investigation medicine has called to her aid other physical sciences. Natural philosophy has furnished the explanation of the mechanical contrivances, and we look to mechanics, hydraulics, and pneumatics to aid us to interpret the phenomena of locomotion, circulation, and respiration. It has furnished in electricity the probable means of interpretation of the force which operates the mechanism, the phenomena of the nerve power; she has furnished the microscope to enable us to see things invisible to the naked sight, whilst chemistry has laid before us the knowledge of the fluids of the body, and enabled us to solve the mysteries of digestion. These sciences have been called the hand-maids of medicine, they are in fact parts of the whole; without them no physiology, without physiology no art of medicine.

But with scalpel, microscope, and chemistry, we fail to delineate the anatomy of the nervous system. It is a truth that, that which is is but the sequence of that which was. The results of to-day could not be had without the investigations of yesterday. In like manner will the knowledge of to-day be the basis of the conclusions of to-morrow-such is the law of intellectual development; such is the sequence of scientific advancement. Sir Charles Bell first showed that the nerves of the human organization are distributed with regularity. It is the basis of the physiology of the nervous system. He first called attention to the functions of the spinal nerves, pointed out their different structures and their different functions; distinguished the sensory and the motor. Marshall Hall succeeded Bell, and we had his excitomotor system. Others succeeded Hall to explain nervous phenomena, among whom stands preëmi-. nent the great Claude Bernard.

But we owe more to Marshall Hall than the explanations he has given of nervous phenomena. $\mathrm{He}$ demonstrated that when the scalpel and the microscope fail, to determine its anatomy, we can yet map out the anatomy of the system. A physiological fact is as certain an indication of nervous distribu- 
tion, as though we could follow its ramifications scalpel in hand or microscope to eye. Observation and experiment is the thread which leads out of the labyrinth, without which we must be lost in its intricate mazes. Marshall Hall has given us a map of the excito-motor system of nerves, Bernard has shown their peripheral distribution. The functions of the brain and spinal marrow are to be laid before us by pursuing the same line of investigation. By experiment, the number of devoted investigators give an earnest promise that the science of life may yet be illustrated, that the opprobrium that medicine is only the empiricism may be expunged, and that the day will yet arrive when medicine will be practised upon laws or principles known and recognized.

The whole forms a beautiful piece of mechanism, whereby under the orders of certain nerve centres it is set in motion. Blood flows in by the arteries regulated by the vaso-motors; the veins receive it, and dilate under the direction of the vaso-dilators; the muscular bands are brought into play by the nerve power conveyed to them, the result being what we call full nutrition. When nerve power is exhausted or its exaltation diminishes, muscles relax, veins contract, the parts are freed from blood. As a typical illustration of voluntary force we will cite its influence on the heart's action--say for seventy yearsand, for convenience, fix the pulsations at sixty per minute. Then we will have the proposition that $60 \times 60=3,600$ beats per hour. Again, 3,600 $\times 10$ $=36,000$ beats in ten hours; or, in the words of the learned Draper, this little organ can execute "three thousand million beats without a stop; and propel a half million tons of blood, and momentarily wasting, repairs its own waste all this time."

The mathematical rhythm of four moving cavities, the perfect closure of its mitral and semi-lunar valves, and the regurgitating play of its tricuspid, have never failed. Much more could be said of other vital organs, under the control of the involuntary force. In the face of all these facts, how strikingly true is the saying, "How fearfully and wonderfully we are made," for, in regard to the heart, we have but to note that its nerve supply springs principally from three little ganglia, and a few nerve fibres originating from the brain, spinal and sympathetic. How delicate the source, how fragile and thread-like the conductors; how readily implicated, how sudden and certain the result. Yet, in death from such a cause, how obscure the character of the lesion!

\section{THE TREATMENT OF DISEASES BY THE HYPODER- MATIC INJECTION OF OILS.'}

BY JOHN V. SHOEMAKER, A.M., M.D.,

OF PHILADEIFHIA.

Experimental and clinical observations have taught us that oils when they cannot be swallowed, or are rejected by the stomach, can be absorbed by inunction and subcutaneous injection; and nu-

1Read before the Section of Practical Medicine, Materia Medica and Therapeutics, at the Thirty-Sixth Annual Meeting of the American Medical Association. merous instances of the absorption and assimilation of oil and fat by the skin have been reported, and their value in certain conditions, employed in this way, has been fully recognised. But the more rapid and effective use of these agents subcutaneously, while known, has had little or no practical application.

Menzel ${ }^{1}$ and Perco first demonstrated the subcutaneous absorption of fat by injecting an ounce of it, in a fluid state, under the skin of a dog, and in the course of forty-eight hours it disappeared, without leaving any local effect. The first practical application of this discovery was probably made by Krueg $^{2}$ on an insane patient who refused to eat. Subcutaneous injections of olive oil, twice daily, were then administered, affording sufficient nourishment to keep the patient in good bodily vigor. At the end of a month he was induced to take his food in the natural way, and the injections were discontinued.

Shortly after this announcement of the practical effects in Krueg's case, a most important result from hypodermatic alimentation in a case of gastric ulcer was reported by Dr. James ' $T$. Whittaker, of Cincinnati, Ohio. According to Dr. Whittakers report, ${ }^{3}$ after exhausting other medication the patient was given subcutaneous injections of drachm doses of milk, alternated with beef extract, every two hours for three days. The temperature declined, the pulse became stronger, and the existing pains and delirium disappeared. The milk and beef extract not being well borne by the skin, cod-liver oil was substituted for them, two drachms being given every two hours for two days, and one day as much as eight ounces was introduced. Two abscesses formed from the milk, but not any ill-effects followed from the oil, which was not only well borne, but its introduction was also free from pain. The inability of some of my patients to bear medicine taken into the stomach induced me, a few years ago, to test thoroughly the subcutaneous absorption of oil, and the effects which followed in some few of the cases under treatment.

Case I.-Constipation.--Miss M., aged 43, was confined to her bed after an operation for cancer; bowels became sluggish, stomach irritable, and all efforts at evacuation failed for six days. On the morning of the seventh day two drachms of castor oil was mixed with an equal quantity of oil of sweet almonds, in order to make the oil sufficiently fluid for use; with a large hypodermatic syringe the injection was deposited into the subcutaneous cellular tissue of the back. An hour afterwards the patient complained of pains through the limbs, which continued to increase until a copious evacuation took place, an hour and a half elapsing from the giving of the injection to the evacuation, and another passage succeeding in a brief period.

Case 2.-Obstinate Constipation,-I was called to see a young man, eighteen years of age, suffering with nausea and vomiting; bowels had not been moved for nine days. Administered the ordinary

\footnotetext{
${ }^{1}$ Wiener medizinische Wochenschrift, April 17, I869.

2Wiener medizinische Wochenschrift, August 21,1875

3 "Hypodermic Alimentation," by Jr. James T. Whittaker, M.D., Cincinnati Clinic, Jan. 22,1876
} 